\title{
Problema Creep-Térmico na Dinâmica de Gases Rarefeitos Baseado no Modelo BGK
}

R.F. KNACKFUSS ${ }^{1}$, I.B. ASEKA ${ }^{2}$, Departamento de Matemática, CCNE, UFSM, 97105-900 Santa Maria, RS, Brasil.

Resumo. Neste trabalho, apresenta-se resultados numéricos para o perfil de velocidade e o perfil de fluxo de calor, relativas ao movimento de um gás rarefeito através de um canal plano sujeito a um gradiente de temperatura. Considera-se, aqui, o canal definido por duas placas paralelas com diferentes constituições químicas, isto é, com coeficientes de acomodação diferentes. Para solucionar este problema, denominado de Creep-Térmico, inicialmente a equação de Boltzmann é aproximada pelas equações cinéticas que, neste caso, são baseadas no modelo BGK. O processo de interação entre o gás e a superfície é descrito pelo modelo de Maxwell e pela condição generalizada de Cercignani-Lampis. A solução do problema é encontrada através de uma versão analítica do método de ordenadas discretas (ADO).

Palavras-chave. Dinâmica de Gases Rarefeitos, Método de Ordenadas Discretas, Modelo de Maxwell, Modelo de Cercignani-Lampis.

\section{Introdução}

Problemas que envolvem dinâmica de gases rarefeitos são frequentemente modelados pela equação de Boltzmann que pode ser aproximada por equações cinéticas. Nestes problemas, as interações dos gases com a superfície têm função importante, visto que, a temperatura da superfície bem como sua rugosidade e sua constituição química influenciam diretamente nestas interações (gás-superfície) conduzindo a diferentes coeficientes de acomodação [5]. Nos modelos matemáticos, as condições de contorno levam em consideração o tipo de superfície através dos núcleos de espalhamento. Dentre os diferentes tipos de núcleo de espalhamento que expressam as diferentes interações entre o gás e a superfície, destacam-se o núcleo de espalhamento de Maxwell [17], que considera a fração $(1-\alpha)$ de partículas refletidas especularmente e o restante $\alpha$ refletida difusivamente e o núcleo de espalhamento de Cercignani-Lampis [6], que diferentemente do núcleo de Maxwell, para uma melhor representação das propriedades físicas da superfície, apresenta dois coeficientes de acomodação: o coeficiente de acomodação do momento tangencial $\left(\alpha_{t}\right)$ e o coeficiente de acomodação da energia cinética devido a componente normal da velocidade $\left(\alpha_{n}\right)$.

\footnotetext{
${ }^{1}$ knackfuss@smail.ufsm.br.

2iaseka@smail.ufsm.br.
} 
Em recentes trabalhos, o núcleo de Cerciganni-Lampis associado ao método de ordenadas discretas analítico (ADO) [2] foi usado para solução de uma classe de problemas baseados nas equações cinéticas $[8,9]$ visando encontrar resultados para análise dos efeitos da superfície, que são importantes em fenômenos que envolvem a dinâmica de gases rarefeitos. Neste sentido, é de suma importância a consideração de diferentes coeficientes de acomodação. Com este objetivo, usa-se, neste trabalho, as condições de contorno de Maxwell e de Cercignani-Lampis (que descrevem a interação gás-superfície) associadas ao método de ordenadas discretas analítico (ADO) [2] para obter a solução do problema Creep-Térmico, baseado no modelo BGK [3], sendo que as placas por onde o gás flui, possuem diferentes constituições químicas.

Vários trabalhos já foram desenvolvidos com o objetivo de resolver problemas da dinâmica de gases rarefeitos com outros métodos, outras equações cinéticas e/ou outros núcleos de interação gás-superfície. Pode-se destacar:

- Barichello et al. [1], onde usa-se o modelo BGK, o método ADO e o núcleo de Maxwell.

- Sharipov [12], onde é usado o modelo $\mathbf{S}$, o método da velocidade discreta e o núcleo de Cercignani-Lampis.

- Garcia e Siewert [7], onde usa-se a equação linearizada de Boltzmann, o método ADO e o núcleo de Cercignani-Lampis.

- Cabrera e Barichello [4], onde é usado o modelo S, o método ADO e o núcleo de Maxwell.

De acordo com Sharipov e Seleznev [13], para o modelo BGK, o número de Prandtl é igual a um. Entretanto, o valor correto desse número é 2/3. A escolha do modelo BGK justifica-se pela possibilidade de estabelecer comparações com futuros trabalhos propostos pelos autores, utilizando outros modelos (S e Gross-Jackson). Além disso, analisar o erro desse modelo com outros modelos que apresentam o número de Prandtl correto.

\section{Formulação Matemática}

Considera-se a equação cinética escrita em termos da perturbação $h(y, \mathbf{c})$ para a função distribuição de uma Maxwelliana local, como

$$
\begin{aligned}
& c_{y} \frac{\partial}{\partial y} h(y, \mathbf{c})+\varepsilon h(y, \mathbf{c})= \\
& \quad \varepsilon \pi^{-3 / 2} \int_{-\infty}^{\infty} \int_{-\infty}^{\infty} \int_{-\infty}^{\infty} e^{-c^{\prime 2}} \boldsymbol{F}\left(\mathbf{c}^{\prime}, \mathbf{c}\right) h\left(y, \mathbf{c}^{\prime}\right) \mathrm{d} c_{x}^{\prime} \mathrm{d} c_{y}^{\prime} \mathrm{d} c_{z}^{\prime}+S(\mathbf{c}),
\end{aligned}
$$

onde o núcleo de espalhamento, para o modelo BGK, é dado por

$$
\boldsymbol{F}\left(\mathbf{c}^{\prime}, \mathbf{c}\right)=1+2\left(\mathbf{c}^{\prime} \cdot \mathbf{c}\right)+(2 / 3)\left(c^{\prime 2}-3 / 2\right)\left(c^{2}-3 / 2\right) .
$$

Além disso, considera-se o caso unidimensional para a variável adimensional y (medida em termos do livre caminho médio $l$ ), as componentes do vetor velocidade 
$\left(c_{x}, c_{y}, c_{z}\right)$ expressas em unidades adimensionais,

$$
S(\mathbf{c})=-c_{x}\left(c_{x}^{2}+c_{y}^{2}+c_{z}^{2}-5 / 2\right) \quad \text { e } \quad \varepsilon=\sigma_{0}^{2} n_{0} \pi^{1 / 2} l,
$$

sendo que $\sigma_{0}$ é o diâmetro de colisão das partículas de gás na aproximação de esferas rígidas e $n_{0}$ é a densidade de equilíbrio das partículas de gás.

Neste trabalho, investiga-se o problema Creep-Térmico para gases rarefeitos entre placas paralelas situadas em $-a$ e $a$. Considera-se dois problemas específicos, ambos descritos pela Eq. (2.1) e diferenciados pelas condições de contorno. Para um dos problemas toma-se as condições de contorno de Maxwell e para o outro as de Cercignani-Lampis [5, 6] que são dadas, respectivamente, por

$$
\begin{aligned}
h\left(\mp a, c_{x}, \pm c_{y}, c_{z}\right)= & \alpha_{l}\left[2 c_{z} u_{w l}+\frac{T_{w}-T_{0}}{T_{0}}\left(c^{2}-2\right)\right]+\left(1-\alpha_{l}\right) h\left(\mp a, c_{x}, \mp c_{y}, c_{z}\right) \\
& +\frac{2 \alpha_{l}}{\pi} \int_{-\infty}^{0} c_{x}^{\prime} \mathrm{d} c_{x}^{\prime} \int_{-\infty}^{\infty} \int_{-\infty}^{\infty} e^{-c^{\prime 2}} h\left(\mp a, c_{x}^{\prime}, \mp c_{y}^{\prime}, c_{z}^{\prime}\right) \mathrm{d} c_{y}^{\prime} \mathrm{d} c_{z}^{\prime},
\end{aligned}
$$

onde $l=1$ representa a placa situada em $-a$ e $l=2$ representa a placa situada em $a, u_{w l}$ é a velocidade da placa e $T_{0}$ é a temperatura constante de referência, e

$$
\begin{aligned}
& h\left(\mp a, c_{x}, \mp c_{y}, c_{z}\right)= \\
& \quad \int_{0}^{\infty} \int_{-\infty}^{\infty} \int_{-\infty}^{\infty} h\left(\mp a, c_{x}^{\prime}, \mp c_{y}^{\prime}, c_{z}^{\prime}\right) R_{l}\left(c_{x}^{\prime}, \mp c_{y}^{\prime}, c_{z}^{\prime}: c_{x}, \pm c_{y}, c_{z}\right) \mathrm{d} c_{x}^{\prime} \mathrm{d} c_{z}^{\prime} \mathrm{d} c_{y}^{\prime},(2.3)
\end{aligned}
$$

sendo

$$
\begin{gathered}
R_{l}\left(c_{x}^{\prime}, c_{y}^{\prime}, c_{z}^{\prime}: c_{x}, c_{y}, c_{z}\right)=\frac{c_{y}^{\prime}}{\pi \alpha_{n_{l}} \alpha_{t_{l}}\left(2-\alpha_{t_{l}}\right)} T_{l}\left(c_{x}^{\prime}: c_{x}\right) S_{l}\left(c_{y}^{\prime}: c_{y}\right) T_{l}\left(c_{z}^{\prime}: c_{z}\right), \\
T_{l}(x: y)=\exp \left[-\frac{\left[\left(1-\alpha_{t_{l}}\right) y-x\right]^{2}}{\alpha_{t_{l}}\left(2-\alpha_{t_{l}}\right)}\right]
\end{gathered}
$$

$\mathrm{e}$

$$
S_{l}(x: y)=\exp \left[-\frac{\left[\left(1-\alpha_{n_{l}}\right)^{1 / 2} y-x\right]^{2}}{\alpha_{n_{l}}}\right] \widehat{I}_{0}\left[\frac{2\left(1-\alpha_{n_{l}}\right)^{1 / 2}|x y|}{\alpha_{n_{l}}}\right] .
$$

Para efeitos computacionais escreve-se

$$
\widehat{I}_{0}(w)=I_{0}(w) \exp (-w)
$$

onde $I_{0}(w)$ é a função de Bessel modificada

$$
I_{0}(w)=\frac{1}{2 \pi} \int_{0}^{2 \pi} \exp (w \cos \phi) \mathrm{d} \phi .
$$

Nota-se que na Eq. (2.4), o núcleo de Cercignani-Lampis inclui dois coeficientes de acomodação definidos como: coeficiente de acomodação da energia cinética devido a componente normal da velocidade $\left(\alpha_{n} \in[0,1]\right)$ e coeficiente de acomodação 
do momento tangencial $\left(\alpha_{t} \in[0,2]\right)[5,6]$. Fisicamente, o núcleo de CercignaniLampis admite a reflexão para trás que pode ocorrer em superfícies rugosas. No caso limite quando $\alpha_{t}=2$ e $\alpha_{n}=0$, a velocidade troca de sinal após uma colisão com uma superfície, trocando, assim, a sua direção. Quando $\alpha_{t}=1$ e $\alpha_{n}=1 \mathrm{o}$ núcleo de Cercignani-Lampis Eq. (2.4) coincide com uma reflexão difusa, por outro lado, no caso limite $\alpha_{t}=0$ e $\alpha_{n}=0$, o mesmo torna-se uma reflexão especular.

Para avaliar as grandezas físicas, segue-se Siewert [15] e admite-se, para o perfil de velocidade

$$
u(y)=\pi^{-3 / 2} \int_{-\infty}^{\infty} \int_{-\infty}^{\infty} \int_{-\infty}^{\infty} e^{-c^{\prime 2}} h\left(y, c_{x}, c_{y}, c_{z}\right) c_{x} \mathrm{~d} c_{x} \mathrm{~d} c_{y} \mathrm{~d} c_{z}
$$

e para o perfil de fluxo de calor

$$
q(y)=\pi^{-3 / 2} \int_{-\infty}^{\infty} \int_{-\infty}^{\infty} \int_{-\infty}^{\infty} e^{-c^{\prime 2}} h\left(y, c_{x}, c_{y}, c_{z}\right)\left(\mathbf{c}^{2}-5 / 2\right) c_{x} \mathrm{~d} c_{x} \mathrm{~d} c_{y} \mathrm{~d} c_{z} .
$$

Tendo em vista a definição das grandezas físicas em termos de momentos da função $h$, multiplica-se, respectivamente, a Eq. (2.1) por

$\phi_{1}\left(c_{x}, c_{z}\right)=\frac{1}{\pi} c_{x} e^{-\left(c_{x}{ }^{2}+c_{z}{ }^{2}\right)} \quad$ e $\quad \phi_{2}\left(c_{x}, c_{z}\right)=\frac{1}{\pi \sqrt{2}} c_{x}\left(c_{x}^{2}+c_{z}^{2}-2\right) e^{-\left(c_{x}{ }^{2}+c_{z}{ }^{2}\right)}$,

integra-se em relação a $c_{x}$ e $c_{z}$, introduz-se a nova notação $\xi=c_{y}$, define-se

$$
h_{1}(y, \xi)=\int_{-\infty}^{\infty} \int_{-\infty}^{\infty} \phi_{1}\left(c_{x}, c_{z}\right) h\left(y, c_{x}, \xi, c_{z}\right) \mathrm{d} c_{x} \mathrm{~d} c_{z}
$$

e

$$
h_{2}(y, \xi)=\int_{-\infty}^{\infty} \int_{-\infty}^{\infty} \phi_{2}\left(c_{x}, c_{z}\right) h\left(y, c_{x}, \xi, c_{z}\right) \mathrm{d} c_{x} \mathrm{~d} c_{z}
$$

e encontra-se as equações

$$
\xi \frac{\partial}{\partial y} h_{1}(y, \xi)+h_{1}(y, \xi)=\pi^{-1 / 2} \int_{-\infty}^{\infty} e^{-\xi^{\prime 2}} h_{1}\left(y, \xi^{\prime}\right) \mathrm{d} \xi^{\prime}-\frac{1}{2}
$$

e

$$
\xi \frac{\partial}{\partial y} h_{2}(y, \xi)+h_{2}(y, \xi)=0
$$

\section{Formulação Vetorial}

Considerando-se $\mathbf{H}(y, \xi)$ um vetor com componentes $h_{1}(y, \xi)$ e $h_{2}(y, \xi)$, pode-se reescrever as Eqs. (2.7) e (2.8) na forma vetorial como

$$
\xi \frac{\partial}{\partial y} \mathbf{H}(y, \xi)+\mathbf{H}(y, \xi)=\int_{-\infty}^{\infty} \mathbf{\Psi}\left(\xi^{\prime}\right) \mathbf{H}\left(y, \xi^{\prime}\right) \mathrm{d} \xi^{\prime}+\mathbf{S}^{*}(\xi),
$$


onde

$$
\begin{gathered}
\boldsymbol{\Psi}\left(\xi^{\prime}\right)=\pi^{-1 / 2} e^{-\xi^{\prime 2}} \mathbf{Q} \\
\mathbf{Q}=\left[\begin{array}{ll}
1 & 0 \\
0 & 0
\end{array}\right], \quad \mathbf{H}(y, \xi)=\left[\begin{array}{c}
h_{1}(y, \xi) \\
h_{2}(y, \xi)
\end{array}\right] \quad \text { e } \quad \mathbf{S}^{*}(\xi)=\left[\begin{array}{c}
-1 / 2 \\
0
\end{array}\right] .
\end{gathered}
$$

A metodologia usada na obtenção da Eq. (3.1) é também aplicada nas condições de contorno. Assim, para as condições de contorno de Maxwell, Eq. (2.2) encontrase, a equação na forma vetorial

$$
\mathbf{H}(\mp a, \pm \xi)=\left(1-\alpha_{l}\right) \mathbf{H}(\mp a, \mp \xi) .
$$

Para as condições de contorno de Cercignani-Lampis, Eq. (2.3) tem-se

$$
\mathbf{H}(\mp a, \pm \xi)=\mathbf{A}_{l} \int_{0}^{\infty} \mathbf{H}\left(\mp a, \mp \xi^{\prime}\right) f_{l}\left(\xi^{\prime}, \xi\right) \mathrm{d} \xi^{\prime},
$$

onde

$$
\mathbf{A}_{l}=\left[\begin{array}{cc}
1-\alpha_{t_{l}} & 0 \\
0 & \left(1-\alpha_{t_{l}}\right)^{3}
\end{array}\right]
$$

e

$$
f_{l}\left(\xi^{\prime}, \xi\right)=\frac{2 \xi^{\prime}}{\alpha_{n_{l}}} \exp \left[-\frac{\left[\left(1-\alpha_{n_{l}}\right)^{1 / 2} \xi-\xi^{\prime}\right]^{2}}{\alpha_{n_{l}}}\right] \widehat{I}_{0}\left[\frac{2\left(1-\alpha_{n_{l}}\right)^{1 / 2} \xi^{\prime} \xi}{\alpha_{n_{l}}}\right],
$$

$\operatorname{com} l=1,2$.

Baseado na notação vetorial, pode-se expressar as grandezas físicas, Eqs. (2.5) e (2.6), como

$$
u(y)=\pi^{-1 / 2} \int_{-\infty}^{\infty} e^{-\xi^{2}}[1 \quad 0] \mathbf{H}(y, \xi) \mathrm{d} \xi
$$

e

$$
q(y)=\pi^{-1 / 2} \int_{-\infty}^{\infty} e^{-\xi^{2}}\left[\left(\xi^{2}-1 / 2\right) \quad \sqrt{2}\right] \mathbf{H}(y, \xi) \mathrm{d} \xi,
$$

que representam, respectivamente, o perfil de velocidade e o perfil de fluxo de calor.

\section{Desenvolvimento da Solução}

O problema definido pela Eq. (3.1) é não-homogêneo, então sua solução é escrita na forma

$$
\mathbf{H}(y, \xi)=\mathbf{H}^{h}(y, \xi)+\mathbf{H}^{p}(y, \xi) .
$$

Para o problema não-homogêneo encontra-se a solução particular

$$
\mathbf{H}^{p}(y, \xi)=\left[\begin{array}{c}
-\frac{1}{2} \xi^{2}+\frac{1}{4} \\
-\frac{1}{\sqrt{2}}
\end{array}\right] .
$$


Substituindo-se a Eq. (4.1) nas Eqs. (3.1), (3.3) e (3.4), conclui-se que a solução homogênea deve satisfazer a equação

$$
\xi \frac{\partial}{\partial y} \mathbf{H}^{h}(y, \xi)+\mathbf{H}^{h}(y, \xi)=\int_{-\infty}^{\infty} \boldsymbol{\Psi}\left(\xi^{\prime}\right) \mathbf{H}^{h}\left(y, \xi^{\prime}\right) \mathrm{d} \xi^{\prime},
$$

as condições de contorno de Maxwell

$$
\mathbf{H}^{h}(\mp a, \pm \xi)-\left(1-\alpha_{l}\right) \mathbf{H}^{h}(\mp a, \mp \xi)=\mathbf{R}_{l}^{*}(\xi)
$$

e as condições de contorno de Cercignani-Lampis

$$
\mathbf{H}^{h}(\mp a, \pm \xi)-\mathbf{A}_{l} \int_{0}^{\infty} \mathbf{H}^{h}\left(\mp a, \mp \xi^{\prime}\right) f_{l}\left(\xi^{\prime}, \xi\right) \mathrm{d} \xi^{\prime}=\mathbf{R}_{l}(\xi),
$$

onde $\boldsymbol{\Psi}\left(\xi^{\prime}\right)$ é dada pela Eq. (3.2),

$$
\mathbf{R}_{l}^{*}(\xi)=\left(1-\alpha_{l}\right) \mathbf{H}^{p}(\mp a, \mp \xi)-\mathbf{H}^{p}(\mp a, \pm \xi)
$$

e

$$
\mathbf{R}_{l}(\xi)=\mathbf{A}_{l} \int_{0}^{\infty} \mathbf{H}^{p}\left(\mp a, \mp \xi^{\prime}\right) f_{l}\left(\xi^{\prime}, \xi\right) \mathrm{d} \xi^{\prime}-\mathbf{H}^{p}(\mp a, \pm \xi) .
$$

Aqui, $\mathbf{A}_{l}$ e $f_{l}\left(\xi^{\prime}, \xi\right)$ são dadas, respectivamente, pelas Eqs. (3.5) e (3.6).

Observa-se que $l$ nas Eqs. (4.3)-(4.6) assume os valores 1 ou 2 representando as paredes do canal.

\subsection{Solução em ordenadas discretas}

Os problemas homogêneos, Eqs. (4.2)-(4.4), são resolvidos através do método de ordenadas discretas analítico. Para isto, define-se um esquema de quadratura e reescreve-se a Eq. (4.1) na versão de ordenadas discretas como

$$
\pm \xi_{i} \frac{d}{d y} \mathbf{H}^{h}\left(y, \pm \xi_{i}\right)+\mathbf{H}^{h}\left(y, \pm \xi_{i}\right)=\sum_{k=1}^{N} \omega_{k} \mathbf{\Psi}\left(\xi_{k}\right)\left[\mathbf{H}^{h}\left(y, \xi_{k}\right)+\mathbf{H}^{h}\left(y,-\xi_{k}\right)\right],
$$

para $i=1,2 \cdots, N$.

Para as Eqs. (4.7) propõe-se as soluções

$$
\mathbf{H}^{h}\left(y, \pm \xi_{i}\right)=\boldsymbol{\Phi}\left(\nu, \pm \xi_{i}\right) e^{-y / \nu}
$$

que substituindo-se na Eq. (4.7) encontra-se

$$
\left(\nu \mp \xi_{i}\right) \boldsymbol{\Phi}\left(\nu, \pm \xi_{i}\right)=\nu \sum_{k=1}^{N} \omega_{k} \boldsymbol{\Psi}\left(\xi_{k}\right)\left[\mathbf{\Phi}\left(\nu, \xi_{k}\right)+\mathbf{\Phi}\left(\nu,-\xi_{k}\right)\right] .
$$

Tem-se, agora, os vetores $\boldsymbol{\Phi}_{+}(\nu)$ e $\boldsymbol{\Phi}_{-}(\nu)$ de dimensão $2 N \times 1$, com $N$ componentes, $2 \times 1$, definidas, respectivamente, por $\boldsymbol{\Phi}\left(\nu, \xi_{\mathbf{i}}\right)$ e $\boldsymbol{\Phi}\left(\nu,-\xi_{\mathbf{i}}\right)$. Após algumas 
manipulações algébricas, encontra-se o problema de autovalores, com autovalores $1 / \nu^{2}$, obtendo-se, assim, os valores da constante de separação $\nu$. Logo, a solução em ordenadas discretas para a equação homogênea é dada por

$$
\begin{aligned}
\mathbf{H}_{ \pm}^{h}\left(y, \pm \xi_{i}\right)= & A_{1} \boldsymbol{\Phi}^{1}+B_{1} \boldsymbol{\Phi}_{ \pm}^{2} \\
& +\sum_{j=2}^{2 N}\left[A_{j} \boldsymbol{\Phi}_{ \pm}\left(\nu_{j}\right) e^{-(a+y) / \nu_{j}}+B_{j} \boldsymbol{\Phi}_{\mp}\left(\nu_{j}\right) e^{-(a-y) / \nu_{j}}\right],
\end{aligned}
$$

onde introduz-se as soluções exatas, $\boldsymbol{\Phi}^{1}$ e $\boldsymbol{\Phi}_{ \pm}^{2}$ de dimensões $2 N \times 1$, definidas, respectivamente, por $N$ (vetores) componentes da forma

$$
\mathbf{F}^{1}=\left[\begin{array}{l}
1 \\
0
\end{array}\right] \quad \text { e } \quad \mathbf{F}_{ \pm}^{2}(y)=\left[\begin{array}{c}
y \mp \xi \\
0
\end{array}\right] .
$$

Agora, com o objetivo de encontrar as grandezas físicas, levando em consideração gases rarefeitos confinados entre placas paralelas com constituições químicas diferentes, não usa-se, aqui, a condição de simetria, $\mathbf{H}(\mathbf{y}, \xi)=\mathbf{H}(-\mathbf{y},-\xi)$, como usada em Knackfuss e Barichello [8]. Este fato ocasiona uma alteração no número de constantes arbitrárias presentes na solução do problema homogêneo, ou seja, analiticamente, obtém-se um sistema algébrico quadrado de ordem $4 N$, diferentemente, do sistema $2 N$ obtido em Knackfuss e Barichello [8].

Para determinar as constantes arbitrárias $A_{j}$ e $B_{j}, j=1, \ldots, 2 N$, para o caso de condições de contorno de Maxwell, substitui-se a Eq. (4.8) na Eq. (4.3) e para o caso das condições de Cercignani-Lampis substitui-se a Eq. (4.8) na Eq. (4.4) obtendo-se dois sistemas de equações algébricas lineares.

Finalmente, encontra-se a solução completa

$$
\begin{aligned}
\mathbf{H}_{ \pm}\left(y, \pm \xi_{i}\right) & =\mathbf{H}_{ \pm}^{p}(y)+A_{1} \boldsymbol{\Phi}^{1}+B_{1} \boldsymbol{\Phi}_{ \pm}^{2} \\
& +\sum_{j=2}^{2 N}\left[A_{j} \boldsymbol{\Phi}_{ \pm}\left(\nu_{j}\right) e^{-(a+y) / \nu_{j}}+B_{j} \boldsymbol{\Phi}_{\mp}\left(\nu_{j}\right) e^{-(a-y) / \nu_{j}}\right] .
\end{aligned}
$$

Aqui, $\mathbf{H}_{ \pm}^{p}(y)=\mathbf{H}^{p}\left(y, \pm \xi_{i}\right)$ denota um vetor $2 N \times 1$ com $N$ componentes $2 \times 1$.

\section{Grandezas Físicas de Interesse}

Para obter as grandezas físicas, na versão em ordenadas discretas, substitui-se a Eq. (4.10) nas Eqs. (3.7) e (3.8).

No que segue usa-se as seguintes definições

$$
\mathbf{N}\left(\nu_{j}\right)=\left[\omega_{1} \boldsymbol{\Lambda}\left(\xi_{1}\right) \quad \omega_{2} \boldsymbol{\Lambda}\left(\xi_{2}\right) \quad \cdots \quad \omega_{N} \boldsymbol{\Lambda}\left(\xi_{N}\right)\right]\left[\boldsymbol{\Phi}_{+}\left(\nu_{j}\right)+\boldsymbol{\Phi}_{-}\left(\nu_{j}\right)\right]
$$

com componentes $N_{1}\left(\nu_{j}\right)$ e $N_{2}\left(\nu_{j}\right)$, onde

$$
\boldsymbol{\Lambda}(\xi)=\pi^{-1 / 2} e^{-\xi^{2}}\left[\begin{array}{cc}
1 & 0 \\
\xi^{2}-1 / 2 & \sqrt{2}
\end{array}\right]
$$


e as expressões

$$
M(y)=A_{j} e^{-(a+y) / \nu_{j}} \quad \text { e } \quad Q(y)=B_{j} e^{-(a-y) / \nu_{j}}
$$

para escrever:

perfil de velocidade

$$
u(y)=A_{1}+B_{1} y+\sum_{j=2}^{2 N}\left[M_{j}(y)+Q_{j}(y)\right] N_{1}\left(\nu_{j}\right) ;
$$

perfil de fluxo de calor

$$
q(y)=-\frac{5}{4}+\sum_{j=2}^{2 N}\left[M_{j}(y)+Q_{j}(y)\right] N_{2}\left(\nu_{j}\right) ;
$$

\section{Resultados Numéricos}

A implementação computacional, para avaliar os resultados numéricos, foi desenvolvida através de programas em linguagem FORTRAN. Para implementar as soluções, inicialmente, define-se o esquema de quadratura associado ao método de ordenadas discretas analítico (ADO). Para muitos problemas na dinâmica de gases rarefeitos, a utilização do procedimento a seguir, tem se mostrado adequado [8, 9]. Objetivando-se calcular as integrais no intervalo $[0, \infty)$, usa-se a transformação nãolinear

$$
u(\xi)=e^{-\xi}
$$

para mapear $\xi \in[0, \infty)$ sob $u \in[0,1]$, e então usa-se o esquema de quadratura de Gauss-Legendre mapeado linearmente no intervalo [0,1]. O próximo passo é a determinação das constantes de separação. Por fim, encontra-se as constantes arbitrárias para, então, obter-se as grandezas físicas de interesse.

Os resultados são apresentados nas Tabelas 1 a 2 e nas Figuras 1 a 3 para diferentes gases, obtidos com $N=60$ pontos de quadratura. Os números entre parênteses que aparecem nestas tabelas representam potências de dez. As notações DE e CL representam, respectivamente, condições de contorno difuso-especular (Maxwell) e condições de contorno de Cercignani-Lampis. Na equação cinética, dada pela Eq. (2.1), o parâmetro de adimensionalização $\varepsilon$ foi considerado arbitrário. No caso do modelo BGK, quando $\varepsilon$ é avaliado em termos da viscosidade ou da condutividade térmica, o seu valor é igual a 1, isto é, $\varepsilon=\varepsilon_{p}=\varepsilon_{t}=1$.

$\mathrm{Na}$ obtenção dos resultados numéricos apresentados nas tabelas e nos gráficos que seguem, considera-se os seguintes gases nobres: Ne (Neônio), Xe (Xenônio) e $\operatorname{Ar}$ (Argônio). Os valores para os coeficientes de acomodação tangencial $\left(\alpha_{t_{1}}\right)$ e coeficiente de acomodação $\left(\alpha_{1}\right)$, para a superfície 1 , são formulados em termos de valores experimentais dados por Lord [10]. Para a superfície 2, os valores dos coeficientes $\left(\alpha_{t_{2}}\right.$ e $\left.\alpha_{2}\right)$ foram reproduzidos de Sharipov [14] que segue o trabalho experimental de Porodnov et al. [11]. 
Em relação ao coeficiente de acomodação normal $\left(\alpha_{n_{1}}\right.$ e $\left.\alpha_{n_{2}}\right)$, acredita-se não existirem resultados experimentais, assim, escolhe-se valores numéri-cos baseados no coeficiente de acomodação térmico dos gases listados acima, apresentados no trabalho de Thomas [16].

Ne: $\alpha_{t_{1}}=0,31, \alpha_{n_{1}}=0,178, \alpha_{t_{2}}=0,849$ e $\alpha_{n_{2}}=0,082$

Xe: $\alpha_{t_{1}}=0,95, \alpha_{n_{1}}=0,77, \alpha_{t_{2}}=1,014$ e $\alpha_{n_{2}}=0,68$

Ar: $\alpha_{t_{1}}=0,67, \alpha_{n_{1}}=0,44, \alpha_{t_{2}}=0,916$ e $\alpha_{n_{2}}=0,222$

Tabela 1: Creep-Térmico: perfil de velocidade $u(y)$, modelo BGK, $2 a=1$

\begin{tabular}{|l|cc|cc|cc|}
\hline \multirow{2}{*}{$y / a$} & \multicolumn{2}{|c|}{$\mathrm{Ne}$} & \multicolumn{2}{c|}{ Xe } & \multicolumn{2}{c|}{$\mathrm{Ar}$} \\
\cline { 2 - 7 } & $\mathrm{DE}$ & $\mathrm{CL}$ & $\mathrm{DE}$ & $\mathrm{CL}$ & $\mathrm{DE}$ & $\mathrm{CL}$ \\
\hline 0,0 & $1,96663(-1)$ & $1,92295(-1)$ & $1,70039(-1)$ & $1,70138(-1)$ & $1,78803(-1)$ & $1,75957(-1)$ \\
0,2 & $1,90684(-1)$ & $1,94052(-1)$ & $1,67501(-1)$ & $1,68025(-1)$ & $1,75256(-1)$ & $1,74686(-1)$ \\
0,4 & $1,81283(-1)$ & $1,92527(-1)$ & $1,60103(-1)$ & $1,61237(-1)$ & $1,67627(-1)$ & $1,69138(-1)$ \\
0,6 & $1,67406(-1)$ & $1,87266(-1)$ & $1,47402(-1)$ & $1,48766(-1)$ & $1,54904(-1)$ & $1,58442(-1)$ \\
0,8 & $1,46528(-1)$ & $1,76846(-1)$ & $1,26036(-1)$ & $1,27908(-1)$ & $1,34502(-1)$ & $1,40219(-1)$ \\
1,0 & $1,05366(-1)$ & $1,53234(-1)$ & $8,12923(-1)$ & $8,39940(-1)$ & $9,25639(-1)$ & $1,01487(-1)$ \\
\hline
\end{tabular}

Tabela 2: Creep-Térmico: perfil de fluxo de calor $q(y)$, modelo BGK, $2 a=1$

\begin{tabular}{|c|cc|cc|cc|}
\hline \multirow{2}{*}{$y / a$} & \multicolumn{2}{|c|}{$\mathrm{Ne}$} & \multicolumn{2}{c|}{ Xe } & \multicolumn{2}{c|}{ Ar } \\
\cline { 2 - 7 } & $\mathrm{DE}$ & $\mathrm{CL}$ & $\mathrm{DE}$ & $\mathrm{CL}$ & $\mathrm{DE}$ & $\mathrm{CL}$ \\
\hline 0,0 & $-9,24828(-1)$ & $-8,49847(-1)$ & $-7,87685(-1)$ & $-7,82379(-1)$ & $-8,49444(-1)$ & $-7,95456(-1)$ \\
0,2 & $-9,06489(-1)$ & $-8,52612(-1)$ & $-7,79281(-1)$ & $-7,75553(-1)$ & $-8,37501(-1)$ & $-7,89891(-1)$ \\
0,4 & $-8,77749(-1)$ & $-8,43552(-1)$ & $-7,56150(-1)$ & $-7,53912(-1)$ & $-8,12911(-1)$ & $-7,69944(-1)$ \\
0,6 & $-8,34752(-1)$ & $-8,20334(-1)$ & $-7,14338(-1)$ & $-7,13625(-1)$ & $-7,71888(-1)$ & $-7,31978(-1)$ \\
0,8 & $-7,68318(-1)$ & $-7,76291(-1)$ & $-6,43387(-1)$ & $-6,44429(-1)$ & $-7,04812(-1)$ & $-6,66169(-1)$ \\
1,0 & $-6,31486(-1)$ & $-6,74377(-1)$ & $-4,87967(-1)$ & $-4,91807(-1)$ & $-5,61409(-1)$ & $-5,20247(-1)$ \\
\hline
\end{tabular}

Para enfatizar os resultados obtidos, reproduz-se abaixo alguns gráficos, Figs. 1 a 3 .

Nas Figs. 1 e 2, observa-se que os resultados não são sensíveis aos coeficientes de acomodação tangencial e normal. Na Fig. 3, nota-se que tanto em 3(a) como em 3(b) há uma similaridade entre as curvas quando usados diferentes núcleos de espalhamento (Maxwell e Cercignani-Lampis), mostrando que os resultados das grandezas físicas não são sensíveis às condições de contorno adotadas, quando considerados $\alpha_{t}=\alpha$. 


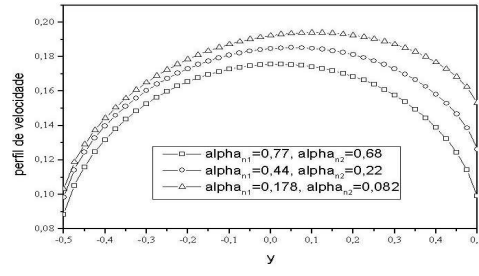

(a) $\alpha_{t 1}=0,31, \alpha_{t 2}=0,849$

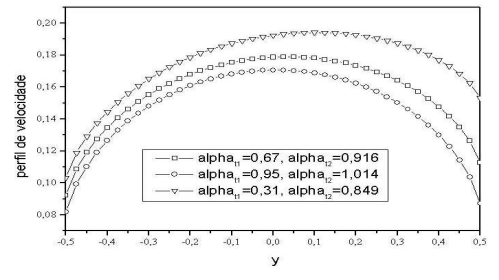

(b) $\alpha_{n 1}=0,178, \alpha_{n 2}=0,082$

Figura 1: Creep-Térmico - Modelo BGK - Condições de Contorno de CercignaniLampis - Perfil de Velocidade, $2 a=1$.

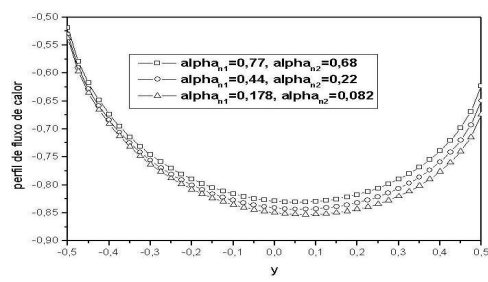

(a) $\alpha_{t 1}=0,31, \alpha_{t 2}=0,849$

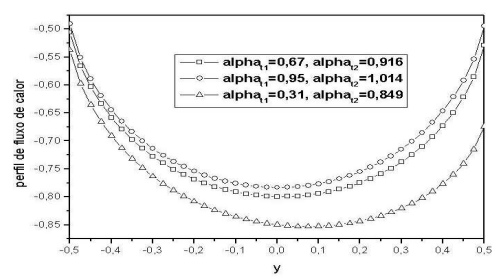

(b) $\alpha_{n 1}=0,178, \alpha_{n 2}=0,082$

Figura 2: Creep-Térmico - Modelo BGK - Condições de Contorno de CercignaniLampis - Perfil de Fluxo de Calor, $2 a=1$.

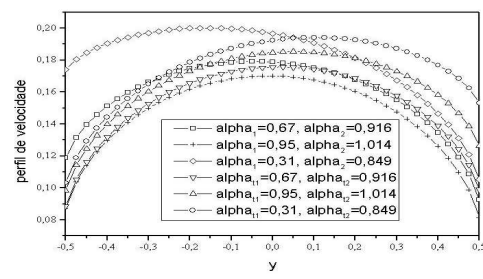

(a) Perfil de Velocidade

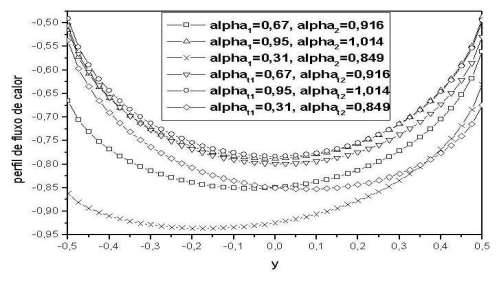

(b) Perfil de Fluxo de Calor

Figura 3: Creep-Térmico - Modelo BGK - Condições de Contorno Difuso Especular e Cercignani-Lampis, $2 a=1, \alpha_{n 1}=0,178$ e $\alpha_{n 2}=0,082$.

\section{Considerações Finais}

A versão analítica do método de ordenadas discretas (ADO), baseado no esquema de quadratura do tipo half-range, foi usado para desenvolver a solução para o problema Creep-Térmico na dinâmica de gases rarefeitos, com a interação gás-superfície 
modelada através do núcleo de Maxwell e de Cercignani-Lampis, considerando-se superfícies com composições químicas diferentes.

Os resultados baseados no modelo BGK com condições de contorno de Maxwell não apresentaram uma diferença significativa quando comparados com os resultados usando as condições de contorno de Cercignani-Lampis.

A condição de simetria não utilizada neste trabalho, torna flexível a análise do comportamento da dinâmica de gases rarefeitos, no sentido de se poder variar os materiais das placas entre as quais o gás flui.

\begin{abstract}
In this work, numerical results are presented for velocity and heat-flow profiles and particle and heat-flow rates of a rarefied gas confined in a plane channel defined by two parallel plates subject to a temperature gradient. The problem is modeled by the BGK kinetic equation and two different types of surface-gas interaction law are analyzed, i. e., the Cercignani-Lampis model that is defined in terms of normal and tangential accommodation coefficients and the usual Maxwell model. Here, the two parallel plates have different chemical compositions, i. e., with different accommodation coefficients. A modern analytical version of the discreteordinates method (ADO) is used to solve this problem and a detailed analysis is performed in regard to the influence of the surfaces on the physical quantities.
\end{abstract}

\title{
Referências
}

[1] L.B. Barichello, M. Camargo, P. Rodrigues, C.E. Siewert, Unified solutions to classical flow problems based on the BGK model, Zeitschrift für Angewandte Mathematik und Physik, 52 (2001), 517-534.

[2] L.B. Barichello, C.E. Siewert, A discrete-ordinates solution for a non-grey model with complete frequency redistribution, Journal of Quantitative Spectroscopy and Radiative Transfer, 62 (1999), 665-675.

[3] P.L. Bhatnagar, E.P. Gross, M. Krook, A model for colission precesses in gas. I. Small amplitude processes in charged and neutral one-component systems, Physics Review, 94 (1954), 511-525.

[4] L.C. Cabrera, L.B. Barichello, Unified solution to some classical problems in rarefied gas dynamics based on the one-dimensional linearized S-model equations, Zeitschrift für Angewandte Mathematik und Physik, 57 (2006), 285-312.

[5] C. Cercignani, "The Boltzmann Equation and its Applications", SpringerVerlag, New York, 1988.

[6] C. Cercignani, M. Lampis, Kinetic models for gas-surface interaction, Transport Theory and Statistical Physics, 1 (1971), 101-114.

[7] R.D.M. Garcia, C.E. Siewert, The linearized Boltzmann equation with Cercignani-Lampis booundary conditions: Basic flow problems in a plane channel, European Journal in Mechanics B/Fluids, 28 (2009), 387-396. 
[8] R.F. Knackfuss, L.B. Barichello, Surface effects in rarefied gas dynamics: an analysis based on the Cercignani-Lampis boundary condition, European Journal of Mechanics B/Fluids, 25 (2006), 113-129.

[9] R.F. Knackfuss, L.B. Barichello, On the temperature-jump problem in rarefied gas dynamics: the effect of the Cercignani-Lampis boundary condition, SIAM - Journal on Applied Mathematics, 66 (2006), 2149-2186.

[10] R.G. Lord, Tangential momentum accommodation coeficients of rare gases on polycrystalline metal surfaces, em "Program Astronautics and Aeronautics Part I", (J.L. Potter, ed.), AIAA, New York, 51 (1977), 531-538.

[11] B.T. Porodnov, P.E. Suetin, S.F. Borisov, V.D. Akinshin, Experimental investigation of rarefied gas flow in different channels, Journal of Fluid Mechanics, 64 (1974), 417-437.

[12] F. Sharipov, Application of the Cercignani-Lampis scattering kernel to calculations of rarefied gas flows. I. Plane flow between two parallel plates, European Journal in Mechanics B/Fluids, 21 (2002), 113-123.

[13] F. Sharipov, V. Seleznev, Data on internal rarefied gas flows, Journal of Physical and Chemical Reference Data, 27 (1998), 657-705.

[14] F. Sharipov, Data on the velocity slip and temperature jump coefficients, em "5th Annual International Conference on Thermal and Mechanical Simulation Experiments in Micro-Electronics and Micro-Systems", pp. 243-249, Brussels, Belgium, 2004.

[15] C.E. Siewert, Generalized boundary conditions for the S-model kinetic equations basic to flow in a plane channel, Journal of Quantitative Spectroscopy and Radiative Transfer, 72 (2001), 75-88.

[16] L.B. Thomas, A collection of some controlled surface thermal accommodation coefficient measurements, em "Rarefied Gas Dynamics", (C.L. Brundin, ed.), Academic Press, New York, (1967) 155-162.

[17] M.M.R. Williams, A review of the rarefied gas dynamics theory associated with some classical problems in flow and heat transfer, Zeitschrift für Angewandte Mathematik und Physik, 52 (2001), 500-516. 